\title{
SPHERICAL, CYLINDRICAL AND ONE-DIMENSIONAL GAS FLOWS*
}

\author{
BY \\ JOSEPH B. KELLER \\ Institute of Mathematical Sciences, New York University
}

\begin{abstract}
The problem of spherical, cylindrical or planar flow of a polutropic gas has been formulated in Lagrangian variables, giving rise to a non-linear second order partial differential equation in two variables, $h$ and $t$. A class of special solutions of this equation has been found by separation of variables, and these solutions depend upon an arbitrary function which is related to the arbitrary entropy distribution in the gas. By specializing this function solutions corresponding to the expansion into a vacuum of an isentropic or non-isentropic gas cloud have been obtained as well as solutions corresponding to the propagation of finite and of strong shocks in variable media.

1. Introduction. Relatively few boundary value problems involving spherical or cylindrical flows of gases or compressible fluids have been solved exactly. In order to solve more problems of this type, particularly those involving variable entropy, we have investigated the flow differential equations, at first without regard to initial or boundary conditions. In this way a class of solutions of the differential equations, depending upon an arbitrary function, has been obtained. In general the solutions of this class represent flows with variable entropy, although some isentropic solutions are included. Then, the arbitrary function is adjusted to satisfy particular initial or boundary conditions. In this way the free expansion into a vacuum of a sphere, cylinder or slab of gas has been treated, as well as the propagation of finite and strong shocks in variable media. The latter treatment includes Primakoff's point-blast solution as a special case. The expansion of a sphere of gas into a vacuum may be of interest to astrophysicists who have treated corresponding one-dimensional problems.

The problem to be solved is formulated in Lagrangian variables, in Sec. 2 of this paper. In Sec. 3 a class of solutions is obtained by the method of separation of variables and in Sec. 4 the isentropic solutions are examined. In Sec. 5 the particle paths of the flows represented by the solutions are analyzed. In Sec. 6 the solutions are used to describe the expansion of a gas into a vacuum, in Sec. 7 to describe strong shock waves propagating in variable media and in Sec. 8 to describe finite shocks in variable media. Section 9 contains conclusions and extensions of the results.
\end{abstract}

2. Formulation. We consider the motion of an inviscid, non-heat-conducting fluid, obeying the polytropic equation of state. The one, two, and three-dimensional cases will be treated together. In the three-dimensional case $y(h, t)$ represents the radius at time $t$ of the particle with the Lagrangian coordinate $h$, which is defined by the equation ${ }^{1}$

$$
h=\int_{\nu(0, t)}^{\nu(h, t)} r^{n-1} \rho(r, t) d r \quad n=1,2,3 .
$$

In the above equation $\rho(r, t)$ is the density at time $t$ and radius $r$, and $n$ is the dimension, which is 3 in the spherical case. In the two dimensional case $y$ represents radial distance from an axis, and in one dimension $y$ is a cartesian coordinate.

*Received June 22, 1955. This work was sponsored by the Office of Naval Research under Contract No. Nonr-285(02).

${ }^{1}$ R. Courant and K. O. Friedrichs, Supersonic flow and shock waves, Interscience Press, 1948, pp. 30-32. 
It is further assumed that all flow variables depend upon $y$ and $t$ only and that flow occurs only in the $y$ direction. This is the assumption of spherical, cylindrical or planar symmetry.

From the definition of $y$, the particle velocity $u$ is given by

$$
u=y_{t} \text {. }
$$

Similarly from (1) the density $\rho$ and specific volume $\tau$ are given by

$$
\tau=\rho^{-1}=y^{n-1} y_{h} .
$$

Because of our assumptions concerning inviscidity and non-conduction, the entropy $s$ of a particle is independent of time (at least between successive shocks). Thus we have

$$
s=s(h) \text {. }
$$

The function $s(h)$ is given by initial data or by shock conditions, and is assumed to be known.

The pressure $p$ is given by the equation of state

$$
p=p(\rho, s)=g(\tau, s) .
$$

For a polytropic gas or liquid

$$
g(\tau, s)=g_{0}+A(s) \tau^{-\gamma} .
$$

The function $A(s)$, the adiabatic exponent $\gamma$, and the internal pressure $g_{0}$ are assumed to be known.

In terms of the above defined quantities, the equation of motion is

$$
y_{t}=-y^{n-1}\left[g_{\tau}\left(y^{n-1} y_{h}\right)_{h}+g_{s} s_{h}\right] .
$$

For a polytropic gas or liquid this becomes, using (6),

$$
y_{\imath t}=\gamma A(s)\left(y^{n-1} y_{h}\right)^{-\gamma-1}\left(y^{n-1} y_{h}\right)_{h} y^{n-1}-A_{h}\left(y^{n-1} y_{h}\right)^{-\gamma} y^{n-1} .
$$

Equation (8) is a second order partial differential equation for $y(h, t)$. The coefficient $A$ is assumed to be a known function of $s$, and by (4), of $h$.

The problem we consider is that of finding solutions of (8). From a particular solution, the flow variables can be found by using (2), (3), (4) and (5).

3. Product solutions. Let us seek product solutions of (8) of the form

$$
y(h, t)=f(h) j(t) .
$$

Inserting (9) into (8), and separating variables we obtain

$$
\begin{gathered}
j^{\prime \prime}-\lambda j^{n(1-\gamma)-1}=0 \\
-A\left[\left(f^{n-1} f^{\prime}\right)^{-\gamma}\right]^{\prime} f^{n-2}-A^{\prime}\left(f^{n-1} f^{\prime}\right)^{-\gamma} f^{n-2}=\lambda .
\end{gathered}
$$

In these equations $\lambda$ is an arbitrary separation parameter and primes represent derivatives with respect to $t$ in (10) and with respect to $h$ in (11).

To solve (10) we multiply by $j^{\prime}$ and integrate, obtaining

$$
\begin{array}{ll}
\left(j^{\prime}\right)^{2}=\frac{2 \lambda}{n(1-\gamma)} j^{n(1-\gamma)}+a & (\gamma \neq 1) \\
\left(j^{\prime}\right)^{2}=2 \lambda \log j+a & (\gamma=1) .
\end{array}
$$


Here $a$ is an integration constant. Thus, unless $j$ is constant, which is possible only if $\lambda=a=0$ or if $j=0$, we have the solution

$$
\begin{array}{ll}
\int_{i_{0}}^{i}\left[\frac{2 \lambda}{n(1-\gamma)} j^{n(1-\gamma)}+a\right]^{-1 / 2} d j=t & (\gamma \neq 1) \\
\int_{i_{0}}^{i}[2 \lambda \log j+a]^{-1 / 2} d j=t & (\gamma=1) .
\end{array}
$$

To solve (11) we differentiate out and obtain

$$
\gamma A\left[f^{\prime \prime} f^{n-1}+(n-1) f^{n-2}\left(f^{\prime}\right)^{2}\right]\left(f^{n-1} f^{\prime}\right)^{-\gamma-1} f^{n-2}-f^{n-2}\left(f^{n-1} f^{\prime}\right)^{-\gamma} A^{\prime}=\lambda .
$$

We now consider the inverse function $h=h(f)$ and denote $h^{\prime}(f)$ by $q(f)$. Then (16) becomes

$\gamma A\left[-q^{\prime} q^{-3} f^{n-1}+(n-1) f^{n-2} q^{-2}\right]\left[f^{n-1} q^{-1}\right]^{-\gamma-1} f^{n-2}-f^{n-2}\left[f^{n-1} q^{-1}\right]^{-\gamma} A^{\prime}(h)=\lambda$.

We shall first treat the case $\gamma \neq 1$ by introducing $z(f)$ and $B(f)$ defined by

$$
z=q^{\gamma-1}, \quad B(f)=A[h(f)] .
$$

With these definitions, (17) becomes (prime denoting differentiation with respect to $f$ )

$$
\begin{aligned}
z^{\prime}+z\left[-(n-1)(\gamma-1) f^{-1}\right. & \left.+\frac{\gamma-1}{\gamma}(\log B)^{\prime}\right] \\
& +\frac{\lambda(\gamma-1)}{\gamma B} f^{(n-1)(\gamma-1)+1}=0 .
\end{aligned}
$$

The solution of (19) is (with $G$ a constant)

$$
z=f^{(n-1)(\gamma-1)} B^{(1-\gamma) / \gamma}\left[G-\frac{\lambda(\gamma-1)}{\gamma} \int^{f} f B^{-1 / \gamma} d f\right] .
$$

Thus we have $q$ from (18) and (20), and finally since $h^{\prime}=q$,

$$
h=\int_{f_{0}}^{f} f^{n-1} B^{-1 / \gamma}\left[G-\frac{\lambda(\gamma-1)}{\gamma} \int^{f} f B^{-1 / \gamma} d f\right]^{\gamma /(\gamma-1)} d f .
$$

Equation (21) gives $f(h)$ implicitly, and thus provides a solution of (11) for $\gamma \neq 1$. The solution may be written more simply by defining $F(f)$ by

$$
F(f)=\left[G-\frac{\lambda(\gamma-1)}{\gamma} \int^{f} f B^{-1 / \gamma} d f\right]^{\gamma /(\gamma-1)}
$$

If $\lambda \neq 0$ this can be solved for $B(f)$ and yields

$$
B(f)=(-\lambda f)^{\gamma}\left(f^{\prime}\right)^{-\gamma} F .
$$

The solution for the flow variables can now be computed from (2)-(5), (9), (21), (22). We have for $\gamma \neq 1$ and $\lambda \neq 0$, remembering that $f=y j^{-1}$ from (9),

$$
\begin{aligned}
u(y, t) & =y j^{\prime} j^{-1} \\
\tau(y, t) & =-\lambda y j^{n-1} / F^{\prime}\left(y j^{-1}\right) \\
p(y, t) & =g_{0}+j^{-n \gamma} F\left(y j^{-1}\right) .
\end{aligned}
$$


Equations (24)-(26) give the flow quantities. In fact, these expressions yield a solution of the Eulerian equations of motion for an arbitrary function $F$ provided that $j(t)$ is given by (14). This is the first main result of this paper. It is to be noted that in these solutions, $u$ is proportional to $y$ and that $F$ must be so chosen that $\tau$, given by (25), is positive. This implies that $F$ must be monotonic in a region where $y$ is of one sign.

In the excluded case $\gamma \neq 1, \lambda=0$ we have instead of (24)-(26) from (2)-(5), (9), and (21)

$$
\begin{array}{ll}
u(y, t)=y j^{\prime} j^{-1} & =y t^{-1}, \\
\tau(y, t)=j^{n} B^{1 / \gamma}\left(y j^{-1}\right) G^{1 /(1-\gamma)} & =t^{n} b\left(y t^{-1}\right), \\
p(y, t)=g_{0}+j^{-n \gamma} G^{\gamma /(1-\gamma)} & =g_{0}+l t^{-n \gamma} .
\end{array}
$$

The new arbitrary function $b$ and arbitrary constant $l$ have been introduced and the expressions simplified by using (14), which gives

$$
j(t)= \pm a^{1 / 2} t .
$$

The origin of $t$ is chosen so that $j(0)=0$.

The corresponding solutions when $\gamma=1$ are obtained from (17) by introducing $B(f)$ as in (18), after which (17) becomes

$$
q^{\prime} q^{-1}-(n-1) f^{-1}+\frac{B^{\prime}}{B}+\frac{\lambda f}{B}=0 .
$$

The solution of $(31)$ is

$$
q(f)=f^{n-1} B^{-1}(f) \exp \int^{f}-\lambda f B^{-1}(f) d f .
$$

Thus

$$
h(f)=\int_{f_{0}}^{f}\left[f^{n-1} B^{-1}(f) \exp \int^{f}-\lambda f B^{-1}(f) d f\right] d f .
$$

Equation (33) yields the solution implicitly. Again we define

$$
F(f)=\exp \int^{f}-\lambda f B^{-1}(f) d f .
$$

Then if $\lambda \neq 0$ we have

$$
B(f)=-\lambda f F\left(F^{\prime}\right)^{-1} .
$$

The solution for the flow variables, if $\gamma=1$ and $\lambda \neq 0$, is again given by (24)-(26) with $\gamma=1$ and $j(t)$ given by (15). Similarly for $\gamma=1$ and $\lambda=0$ the solution is given by (27)-(29) with $\gamma=1, G=1$ and $j(t)$ given by (30).

Equations (24)-(26) and (27)-(29) represent non-isentropic solutions of the flow equations depending upon an arbitrary function. In order to construct these solutions explicitly one need merely evaluate the integrals in (14) or (15).

4. Isentropic case. The above solution can be specialized to the isentropic case by setting $A=B=$ a constant. Then from (22) and (34) we obtain

$$
\begin{array}{ll}
F(f)=\left[G-\frac{\lambda(\gamma-1)}{2 \gamma B^{1 / \gamma}} f^{2}\right]^{\gamma /(\gamma-1)} & \gamma \neq 1, \\
F(f)=\exp \frac{-\gamma f^{2}}{2 B} & \gamma=1 .
\end{array}
$$


Thus the solutions given by (24)-(26) become, for $\gamma \neq 1$ and $\lambda \neq 0$

$$
\begin{aligned}
& u(y, t)=y j^{\prime} j^{-1}, \\
& \tau(y, t)=j^{n} B^{1 / \gamma}\left[G-\frac{\lambda(\gamma-1)}{2 \gamma B^{1 / \gamma}} y^{2} j^{-2}\right]^{-1 /(\gamma-1)}, \\
& p(y, t)=g_{0}+j^{-n \gamma}\left[G-\frac{\lambda(\gamma-1)}{2 \gamma B^{1 / \gamma}} y^{2} j^{-2}\right]^{\gamma /(\gamma-1)} .
\end{aligned}
$$

In these solutions $B$ and $G$ are constants and $j$ is given by (14). For $\lambda=0$, and all $\gamma$ the solution (27)-(29) applies with $b$ constant.

For $\gamma=1$ and all $\lambda,(38)$ is unchanged, but the other equations become

$$
\begin{aligned}
& \tau(y, t)=j^{n} B \exp \frac{-\lambda j^{-2} y^{2}}{2 B}, \\
& p(y, t)=g_{0}+j^{-n} \exp \frac{\lambda j^{-2} y^{2}}{2 B} .
\end{aligned}
$$

Here $B$ is a constant and $j$ is given by (15).

As an example of these isentropic solutions, let $j(t)$ be given by (48) and assume $G=0$ in (39) and (40). Then (38)-(40) yield the power solutions (if $\gamma \neq 1, n(\gamma-1) / 2$ $\neq-1$

$$
\begin{aligned}
u(y, t) & =\frac{2}{n(\gamma-1)+2} y t^{-1} \\
\tau(y, t) & =\left[\frac{\gamma B}{n}\left(\frac{n(\gamma-1)+2}{\gamma-1}\right)^{2}\right]^{1 /(\gamma-1)}\left(y t^{-1}\right)^{-2 /(\gamma-1)}, \\
p(y, t) & =g_{0}+B\left[\frac{\gamma B}{n}\left(\frac{n(\gamma-1)+2}{\gamma-1}\right)^{2}\right]^{\gamma /(1-\gamma)}\left(y t^{-1}\right)^{2 \gamma /(\gamma-1)} .
\end{aligned}
$$

5. Particle paths. A particle designated by a fixed value of $h$, follows the path given by (9), i.e. $y=f(h) j(t)$ where $j(t)$ is a solution of (12) if $\gamma \neq 1$ and of (13) if $\gamma=1$. Let us first consider the case in which the constant $\lambda$ in (12) and (13) is zero. Then in both cases we have

$$
j(t)=j(0) \pm a^{1 / 2} t .
$$

If the origin of $t$ is shifted so that $j(0)=0$ then this becomes

$$
j(t)= \pm a^{1 / 2} t .
$$

This solution has already been used to simplify (27)-(29), [see Eq. (30)]. In the motion described by (44) with the plus sign, all the particles start from the origin at $t=0$ and each moves outward with the constant speed $a^{1 / 2} f(h)$. With the minus sign all particles move toward the origin, each with the constant speed $-a^{1 / 2} f(h)$ and they all arrive there at $t=0$.

Now let us examine the case $\gamma>1, \lambda>0$. Then in order that $\left(j^{\prime}\right)^{2}$ in (12) be positive, we must have the constant $a$ in (12) positive. From (12) we see that the solution is then 
of the form shown in Fig. 1 when the origin of $t$ is chosen so that $j^{\prime}(0)=0$. This choice of origin requires that the constant $a=-2 \lambda j_{0}^{n(1-\gamma)} /[n(1-\gamma)]$.

This solution is even in $t$. Each particle has the constant speed $-a^{1 / 2} f(h)$ at $t=-\infty$ and slows down until it comes to momentary rest at $t=0$ with $y=f(h) j(0)$, after which

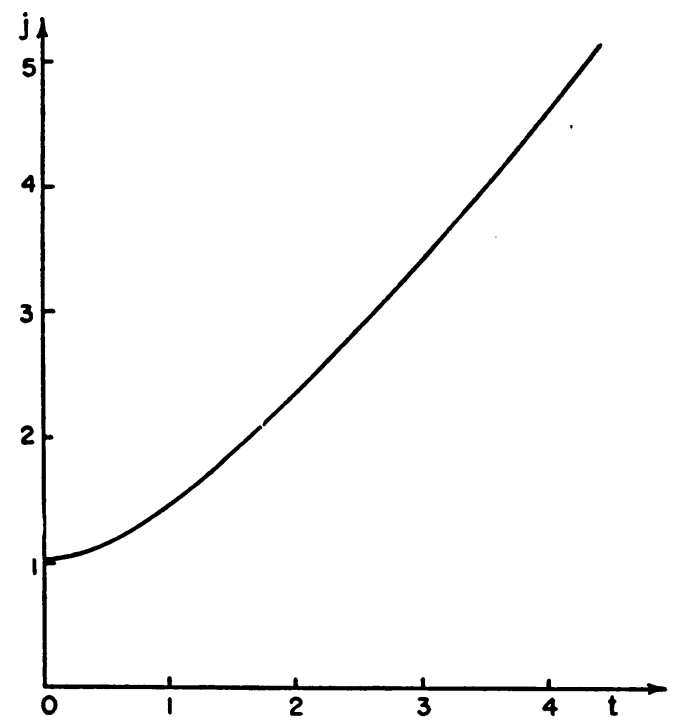

Fra. 1. The behavior of $j(t)$ for $\gamma>1, \lambda>0$. The origin of $t$ is so chosen that $j^{\prime}(0)=0$ which requires $a=-2 \lambda j(0)^{n(1-\gamma)} /[n(\gamma-1)]$. At $t \rightarrow \pm \infty, j^{\prime} \rightarrow \mp a^{1 / 2}$. The curve is obtained from Eq. (14) with $\gamma=1.4$, $n=3, \lambda=j(0)=1$.

it again moves outward ultimately approaching the speed $+a^{1 / 2} f(h)$. The previous solution in which $j(0)=0$ is obviously a limiting form of this solution. If we only consider that half the solution $t \geq 0$ we have the expansion of a gas initially at rest.

The other possibility when $\gamma>1$ is that $\lambda<0$. The nature of the solution now depends upon the sign of $a$ as is shown in Fig. 2 which is obtained by analyzing (12). In the first case $(a>0)$ each particle has the constant speed $-a^{1 / 2} f(h)$ at $t=-\infty$ and speeds up until it reaches the origin at $t=0$ with infinite speed. Thus all the gas collapses into the origin. The positive branch of the curve describes the reverse phenomenon -all the gas is released from the origin at $t=0$ with infinite speed and it spreads out and slows down, each particle approaching the constant speed $a^{1 / 2} f(h)$ as $t$ becomes infinite. The second case $(a=0)$ is similar to the first, but the speed approached as $t$ approaches plus or minus infinity is zero. The explicit solution in this case is

$j(t)=\left\{\left[\frac{2 \lambda}{n(1-\gamma)}\right]^{1 / 2}\left(\frac{n(\gamma-1)+2}{2}\right) t\right\}^{2 /[n(\gamma-1)+2]}$

$$
\text { if } \gamma \neq 1, a=0, \frac{1}{2} n(\gamma-1) \neq-1 \text {. }
$$

The third case $(a<0)$ is different from the preceding cases. The solution is periodic, all the gas being released from the origin at some instant, expanding, coming to rest, collapsing into the origin again, etc.

When $\gamma=1$ and $\lambda<0$ the solution has, for all values of $a$, the behavior shown in Fig. 2c while for $\lambda>0$ it has the behavior shown in Fig. 1, as can be seen from (13). 

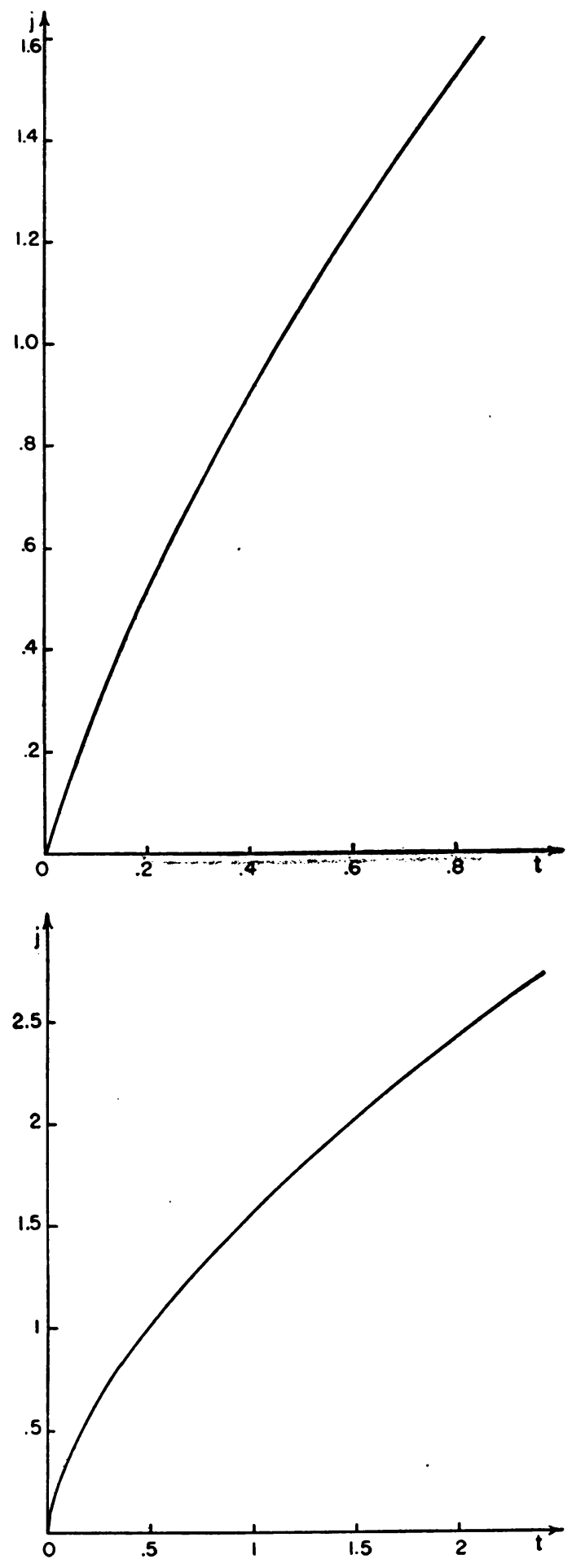

Figs. 2a and b. (For legend see p. 178.) 


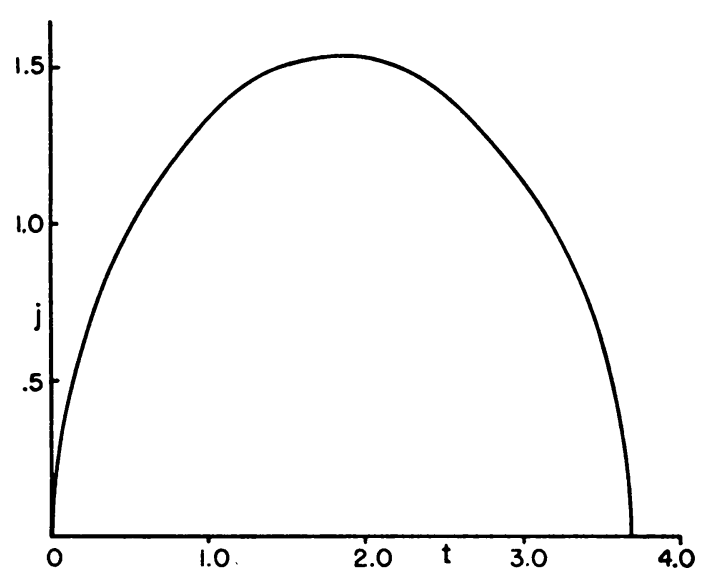

Fig. 2c. The behavior of $j(t)$ for $\gamma>1$ and $\lambda>0$. The origin of $t$ is so chosen that $j(0)=0$. In the first case, $a>0, j^{\prime}$ approaches $\mp a^{1 / 2}$ as $t \rightarrow \pm \infty$. In the second case $a=0, j^{\prime}$ approaches zero as $t \rightarrow \pm \infty$, and in the third case $a<0, j$ is periodic in $t$. The curves are computed for $\gamma=1.4, n=3, \lambda=-1, a=1$ in the first case and $a=-1$ in the third case, using Eq. (14). In the second case $a=0$, the solution is given by (48).

6. Application: Free expansion of gas into vacuum. Let us apply the isentropic solution (38-40) to the free expansion into a vacuum of a gas with $\gamma>1$. Then the pressure must be zero for some value of $h$, which represents the interface between gas and vacuum. If the interface is initially at $y=y_{0}$, and if $g_{0}=0$ since the medium is a gas, we need merely set $G=[\lambda(\gamma-1)] y_{0}^{2} j(0)^{-2} / 2 \gamma B^{1 / \gamma}$ in $(39,40)$. Then $p=0$ at the interface which is given by

$$
y=y_{0} \frac{j(t)}{j(0)} .
$$

The solution $j(t)$ which must be taken is of the type shown in Fig. 1 , if $y_{0}$ and $j(0)$ are not zero. Thus the gas is initially at rest and, according to (40), the initial pressure decreases from a maximum at the center $y=0$ to zero at $y=y_{0}$ as shown in Fig. 3. Then the gas accelerates outward, each particle approaching a constant speed while the speed at any instant is proportional to $y$. The pressure at any particle varies as the $-n \gamma$ power of its radius, thus decreasing as the radius increases. The preceding solution applies to the expansion into a vacuum of a sphere $(n=3)$, a cylinder $(n=2)$ or a slab $(n=1)$ of gas initially at rest, and with uniform entropy.

In a similar manner, but by using solutions for $j(t)$ of the types shown in Figs. 2a and $2 \mathrm{~b}$, solutions describing the expansion of an isentropic gas which fills all space except an expanding region of vacuum around the origin (i.e. a hole), can be constructed.

For a gas with $\gamma=1$ which is also isentropic, it is possible to construct the solution for an expanding gas cloud which fills all space initially, but which has a Gaussian distribution of density. To this end we choose $\lambda>0$, in which case $j(t)$ has the form shown in Fig. 1. Then $u, t$, and $p$ are given by $(38,41,42)$, with $g_{0}=0$ in (42) if the medium is a gas. This solution describes a gas cloud initially at rest, which subsequently expands, the velocity of all particles ultimately approaching the same constant value. If this solution is also considered for $t<0$ it describes a gas cloud which contracts, comes to rest and expands again.

Another interesting motion of a gas with any value of $\gamma$ results if (30) is used for 
$j(t)$ with the plus sign, and if $\lambda=0$ in $(39,40)$. We then obtain, setting $g_{0}=0$ in (40) to describe a gas,

$$
\begin{aligned}
u & =y t^{-1}, \\
\tau & =a^{n / 2} B t^{n}, \\
p & =a^{-n \gamma / 2} B^{1-\gamma} t^{-n \gamma} .
\end{aligned}
$$

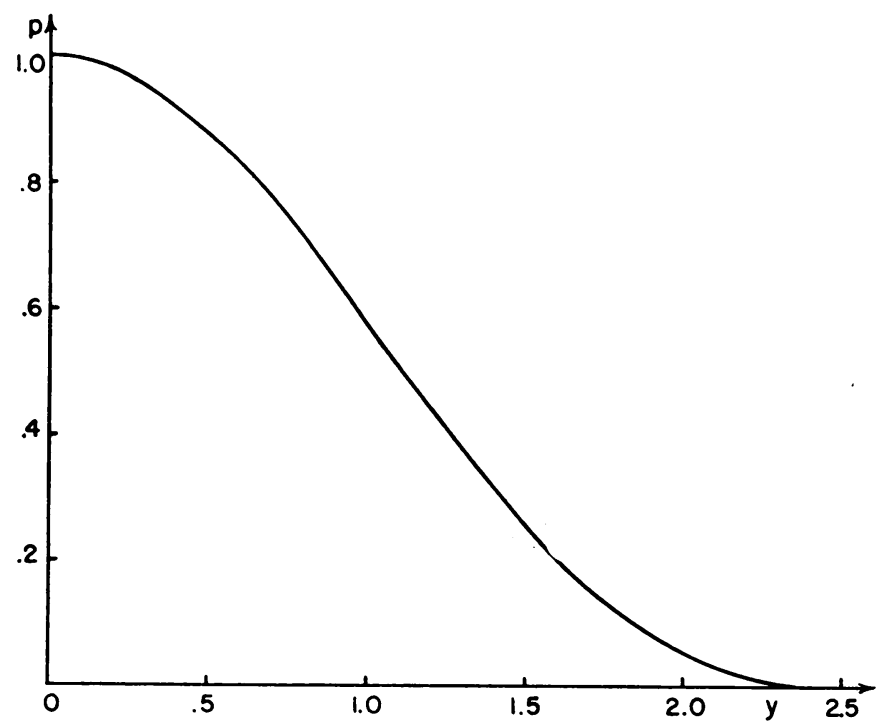

Fig. 3. Initial pressure distribution in an expanding spherical, cylindrical or planar gas cloud, computed from Eq. (40) with $g_{0}=0, \gamma=1.4, G=B=j=1, \lambda=1$. The same curve applies at later times if the vertical scale refers to $p j^{n \gamma}$ and the horizontal scale to $y j^{-1}$.

This solution represents the expansion (or contraction if $t$ is replaced by $-t$ ) of a gas of uniform density, pressure and entropy which fills space.

Similar motions of gases in which the entropy is not initially constant can also be constructed by using equations (24-26) to describe the flow. In order that $p$ equal zero at the interface between gas and vacuum, $F$ must be chosen to vanish at some value of its argument, which value will then correspond to the interface. Aside from this condition, $F$ may be chosen arbitrarily.

7. Application: Strong shocks in variable media. Let us suppose that a shock given by the equation $y=R(t)$ moves into a variable medium of density $\rho_{0}(y)$, pressure $p_{0}(y)$ and velocity zero. The pressure $p$, velocity $u$ and density $\rho$ just behind the shock are related to the corresponding quantities in front of it by the shock conditions. These conditions are

$$
\begin{gathered}
\frac{\rho}{\rho_{0}}=\frac{(\gamma+1) p+(\gamma-1) p_{0}}{(\gamma-1) p+(\gamma+1) p_{0}} \approx \frac{\gamma+1}{\gamma-1}, \quad(\gamma \neq \pm 1) \\
u=\frac{2\left(p-p_{0}\right)}{\left\{2 \rho_{0}\left[(\gamma+1) p+(\gamma-1) p_{0}\right]\right\}^{1 / 2}} \approx\left[\frac{2 p}{(\gamma+1) \rho_{0}}\right]^{1 / 2}, \\
R^{\cdot}=\left[\frac{(\gamma+1) p+(\gamma-1) p_{0}}{2 \rho_{0}}\right]^{1 / 2} \approx\left[\frac{(\gamma+1) p}{2 \rho_{0}}\right]^{1 / 2} .
\end{gathered}
$$

The second expressions on the right apply to a strong shock, for which $p \gg p_{0}$. 
We shall now assume that the flow behind the shock is a "product" solution given by (24)-(26), and that the shock is strong. We wish to determine the functions $F\left(y j^{-1}\right)$, $R(t), \rho_{0}(y)$ and $p_{0}(y)$ for which such a solution is possible. To this end, we insert (24)-(26) into (53)-(55) and obtain

$$
\begin{gathered}
\frac{F^{\prime}\left(R j^{-1}\right)}{-\lambda R j^{n-1} \rho_{0}(R)}=\frac{\gamma+1}{\gamma-1} \\
\frac{R j^{\prime}}{j}=\left[\frac{2 g_{0}+2 j^{-n \gamma} F\left(R j^{-1}\right)}{(\gamma+1) \rho_{0}(R)}\right]^{1 / 2} \\
R^{\cdot}=\left[\frac{(\gamma+1)\left\{g_{0}+j^{-n \gamma} F\left(R j^{-1}\right)\right\}}{2 \rho_{0}(R)}\right]^{1 / 2} .
\end{gathered}
$$

From (57) and (58) we have

$$
\frac{R^{\cdot}}{R}=\frac{(\gamma+1)}{2} \cdot \frac{j^{\prime}}{j} .
$$

Solving, and introducing the integration constant $R_{0}$, we have for $R(t)$ the expression

$$
R(t)=R_{0}[j(t)]^{(\gamma+1) / 2} .
$$

Inserting (60) into (57) and making use of (12), satisfied by $j(t)$, we find

$$
\begin{aligned}
F(x)=\frac{\gamma+1}{2} \rho_{0}\left(x^{(\gamma+1) /(\gamma-1)} R_{0}^{2 /(1-\gamma)}\right) \cdot\left[\frac{2 \lambda}{n(1-\gamma)}\left(x R_{0}^{-1}\right)^{-2 n}+D\right] \\
\cdot R^{(-2 n \gamma) /(\gamma-1)} x^{[(2 n+2) \gamma-21 /(\gamma-1)}-g_{0}\left(x R_{0}^{-1}\right)^{(2 n \gamma) /(\gamma-1)} .
\end{aligned}
$$

Thus $R$ is determined by (60), $F$ is related to $\rho_{0}$ by (61), and (57), (58) are satisfied, although the constants $R_{0}, D$, and $\lambda$ are still arbitrary. We now insert (60), (61) into (56) to determine $F$. We obtain, if $g_{0}=0$,

$$
F(x)=F_{0} x^{n}\left[\frac{2 \lambda R_{0}^{2 n}}{n(1-\gamma)}+D x^{2 n}\right]^{-1 / 2} .
$$

From (61) and (62) we then find an expression for $\rho_{0}(R)$, namely

$$
\begin{aligned}
\rho_{0}(R)=\frac{2 F_{0} R_{0}^{(2 n \gamma) /(\gamma-1)}}{\gamma+1}\left[\frac{2 \lambda R_{0}^{2 n}}{n(1-\gamma)}+D R_{0}^{(4 n) /(\gamma+1)} R^{(2 n(\gamma-1) 1 /(\gamma+1)}\right]^{-3 / 2} \\
\cdot R^{[(n-2) \gamma-3 n+2] /(\gamma+1)} R^{(2(n-2) \gamma-6 n+4] / 1(\gamma+1)(\gamma-1)]} .
\end{aligned}
$$

In (62) and (63) $F_{0}$ is an arbitrary constant. The corresponding results with $g_{0} \neq 0$ are somewhat more complicated.

We have thus obtained a solution with a strong shock moving into a variable medium at rest, with density given by (63). The constants $F_{0}, R_{0}, \lambda$ and $D$ are arbitrary in this equation, but only two essential combinations of these constants occur. The shock curve is given by (60), and $j(t)$ by (14). The flow is given by (24)-(26) with $F(x)$ given by (62). The flow might be produced by a piston following one of the particle paths

$$
Y(t)=Y_{0} j(t) .
$$

The solution was deduced for $\gamma \neq \pm 1$ and $\lambda \neq 0$. 
As an example of these solutions, let us suppose that $\rho_{0}(R)=$ constant. From (63) we find that this requires

$$
D=0, \quad \gamma=\frac{3 n-2}{n-2} .
$$

The last condition can be fulfilled only for $n=3$, in which case $\gamma=7$; $(n=1$ leads to $\gamma=-1$, which was excluded in the derivation, and $n=2$ yields no value of $\gamma$ ). In this case we have from (14), (62)

$$
j=\left[\frac{5(-\lambda)^{1 / 2}}{3} t\right]^{1 / 10}, \quad F(x)=x^{3} \frac{3 F_{0} R_{0}^{-n}}{(-\lambda)^{1 / 2}} .
$$

The solution computed by using (66) in (24)-(26) (with $\lambda<0$ ) is exactly the point blast solution of Taylor's type first found by $\mathrm{H}$. Primakoff, and applicable to high energy explosions in water. [See ${ }^{1}$, p. 424.]

8. Application: Finite shocks in variable media. We may also apply the above method to determine finite shocks in variable media. Then we must satisfy the exact shock conditions (53)-(55) rather than the strong shock conditions. Inserting (24)-(26) into (53)-(55) yields with $g_{0}=0$

$$
\begin{gathered}
\frac{F^{\prime}(x)}{-\lambda R j^{n-1} \rho_{0}}=\frac{(\gamma+1) j^{-n \gamma} F+(\gamma-1) p_{0}}{(\gamma+1) p_{0}+(\gamma-1) j^{-n \gamma} F} \quad x=R j^{-1} \\
R j^{\prime} j^{-1}=\frac{j^{-n \gamma} F-p_{0}}{\left(\rho_{0} / 2\right)^{1 / 2}\left[(\gamma+1) j^{-n \gamma} F+(\gamma-1) p_{0}\right]^{1 / 2}}, \\
R^{\cdot}=\frac{1}{\left(2 \rho_{0}\right)^{1 / 2}}\left[(\gamma+1) j^{-n \gamma} F+(\gamma-1) p_{0}\right]^{1 / 2}
\end{gathered}
$$

Equations (67)-(69) are a set of two first order ordinary differential equations and one algebraic equation for the determination of the four functions $F(x), R(t), p_{0}(R)$ and $\rho_{0}(R)$. Equation (14) gives $j(t)$. This system is evidently under-determined and will have infinitely many solutions. In the strong shock case, however, $p_{0}$ did not occur and thus the system was determined.

To solve the above system we could impose some relation between $p_{0}$ and $\rho_{0}$ and eliminate both these functions by means of that relation and (68). A pair of simultaneous first order equations for $F(x)$ and $R(t)$ would result. However, these equations can be treated separately since, from (68) and (69) we have by eliminating $F$,

$$
R \cdot=\frac{\gamma+1}{4} R j^{\prime} j^{-1} \pm\left[\frac{(\gamma+1)^{2}}{16}\left(R j^{\prime} j^{-1}\right)^{2}+\gamma p_{0} \rho_{0}^{-1}\right]^{1 / 2} .
$$

This is an equation for $R(t)$ if the ratio $p_{0} \rho_{0}^{-1}$ is given as a function of $R$. (Equation (70) also holds when $g_{0} \neq 0$.) After solving this, (67) can be solved for $F$, and then $p_{0}$ and $\rho_{0}$ can be obtained.

We will now investigate the shock curve $R(t)$ given by (70), in the special case in which $j(t)$ is given by (48) with $C=0$ and $\gamma p_{0} \rho_{0}^{-1}=c_{0}^{2}$ is constant. The latter assumption means that the temperature ahead of the shock is constant. Making use of (48), (70) becomes

$$
R^{\cdot}=\frac{\gamma+1}{2[n(\gamma-1)+2]} R t^{-1} \pm\left[\frac{(\gamma+1)^{2}}{4[n(\gamma-1)+2]^{2}}\left(R t^{-1}\right)^{2}+c_{0}^{2}\right]^{1 / 2}
$$


If we now introduce $U(t)=R t^{-1}$ in (71) we obtain an equation for $U$ in which the variables separate. One solution is the constant solution

$$
U= \pm c_{0}\left(1-\frac{\gamma+1}{n(\gamma-1)+2}\right)^{-1 / 2} .
$$

In this case, which is physically possible only when $(\gamma+1) /[n(\gamma-1)+2]<1$, the shock curve is the straight line

$$
R=U t= \pm c_{0}\left(1-\frac{\gamma+1}{n(\gamma-1)+2}\right)^{-1 / 2} t
$$

When $U$ does not have the value given by (72), the equation for $U$ yields

$$
\begin{aligned}
\int_{U_{0}}^{U(t)}\left\{\left(\frac{\gamma+1}{2 n(\gamma-1)+4}-1\right) U\right. & \\
& \left. \pm\left[\frac{(\gamma+1)^{2}}{[2 n(\gamma-1)+4]^{2}} U^{2}+c_{0}^{2}\right]^{1 / 2}\right\}^{-1} d U=\log \frac{t}{t_{0}} .
\end{aligned}
$$

Integrating (74) yields

$$
b t=| \pm a+(a-1) \cos \theta|^{(1-a) /(2 a-1)}\left(\sin \frac{\theta}{2}\right)^{-1 /(a \pm a-1)}\left(\cos \frac{\theta}{2}\right)^{1 /(+a-a+1)} .
$$

Here $\theta=\cot ^{-1}\left[a U / c_{0}\right], a=(\gamma+1) /[2 n(\gamma-1)+4]$ and $b$ is a constant. Thus we have

$$
R=U t=\frac{c_{0}}{a b} \cdot b t \cot \theta .
$$

Since $b t$ is given in terms of $\theta$ by (75), $R(t)$ is given parametrically in terms of $\theta$. A graph of $\left[(a b) / c_{0}\right] R$ versus $b t$ is given in Fig. 4 for $n=3$ and $\gamma=1.4$, which represents an expanding spherical shock in air. When the minus sign is chosen in (71)-(75) the same curves are obtained as with the plus sign, provided $t$ is replaced by minus $t$, thus representing a converging shock. Of course, only one of any such pair of solutions can occur physically, since only one obeys the entropy inequality at the shock.

For small vălues of $t$ we have

$$
R \approx K t^{2 a}
$$

where $K$ is a constant. For $\gamma=7$ and $n=3$ we find $a=1 / 5$ and thus $R \sim t^{2 / 5}$, which is the behavior of Primakoff's point blast solution for small $t$. For large $t, R$ behaves linearly in $t$, as in (73).

To determine $p_{0}$ and $F$, we have from (69) and the condition $\gamma p_{0} \rho_{0}^{-1}=c_{0}^{2}$,

$$
F=\frac{2 \rho_{0} R^{\cdot 2}-(\gamma-1) p_{0}}{(\gamma+1) j^{-n \gamma}}=\frac{2 \gamma p_{0}}{(\gamma+1) c_{0}^{2}} R^{\cdot 2} j^{n \gamma}-\frac{\gamma-1}{\gamma+1} p_{0} j^{n \gamma} \text {. }
$$

Thus, if dot denotes time derivative and prime denotes derivative of a function with respect to its argument, we have

$$
\begin{aligned}
F^{\cdot}= & \frac{2 \gamma}{(\gamma+1) c_{0}^{2}}\left(p_{0}^{\prime} R^{\cdot 3} j^{n \gamma}+2 p_{0} R \cdot R^{\cdot \cdot j^{n \gamma}}+n \gamma p_{0} R^{\cdot 2} j^{n \gamma-1} j^{\prime}\right) \\
& \quad-\frac{\gamma-1}{\gamma+1}\left(p_{0}^{\prime} R^{\cdot} j^{n \gamma}+n \gamma p_{0} j^{n \gamma-1} j^{\prime}\right) \\
= & F^{\prime}\left(R^{\cdot} j^{-1}-R j^{-2} j^{\prime}\right) .
\end{aligned}
$$




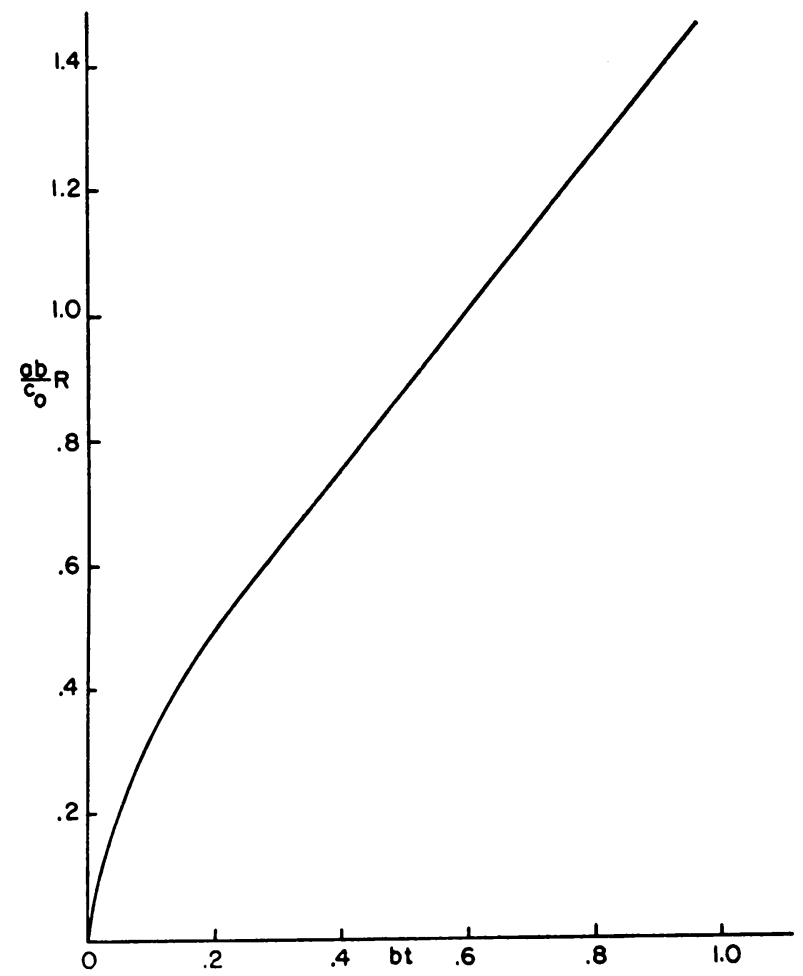

FIG. 4. The radius $R$ of an expanding spherical shock in air as a function of time $t$ computed from the parametric equations $(75,76)$ with $n=3, \gamma=1.4$.

Using (78) and (79) in (67) yields the following equation for $p_{0}$

$$
\begin{gathered}
{\left[\frac{2 \gamma}{(\gamma+1) c_{0}^{2}}\left(p_{0}^{\prime} R^{\cdot 3} j^{n \gamma}+2 p_{0} R \cdot R^{\cdot \cdot} j^{n \gamma}+n \gamma p_{0} R^{\cdot 2} j^{n \gamma-1} j^{\prime}\right)\right.} \\
\left.-\frac{\gamma-1}{\gamma+1}\left(p_{0}^{\prime} R \cdot j^{n \gamma}+n \gamma p_{0} j^{n \gamma-1} j^{\prime}\right)\right]\left[-\lambda R j^{n-1} \gamma p_{0} c_{0}^{-2}\left(R j^{-1}-R j^{2} j^{\prime}\right)\right]^{-1} \\
=2 \gamma c_{0}^{-2} R^{\cdot 2}\left[\gamma+1+\frac{(\gamma-1) 2 \gamma R^{\cdot 2}}{(\gamma+1) c_{0}^{2}}-\frac{(\gamma-1)^{2}}{(\gamma+1)}\right]^{-1} .
\end{gathered}
$$

Lpon introducing $p_{0}^{\cdot}=p_{0}^{\prime} R^{*}$, the above equation can be integrated yielding $p_{0}[R(t)]$ as a function of $t$. To obtain $p_{0}(R)$, we must insert $t=t(R)$ from (75) and (76) into this result. Then when $p_{0}[R(t)]$ is known, $F$ can be found from (78). These calculations will not be carried out here.

9. Conclusion and extensions. The problem of spherical, cylindrical or planar flow of a polytropic gas has been formulated in Lagrangian variables, giving rise to a nonlinear second order partial differential equation in two variables, $h$ and $t$. A class of special solutions of this equation has been found by separation of variables, and these solutions depend upon an arbitrary function which is related to the arbitrary entropy distribution in the gas. By specializing this function solutions corresponding to the expansion into a vacuum of an isentropic or non-isentropic gas cloud have been obtained 
as well as solutions corresponding to the propagation of finite and of strong shocks in variable media.

Other types of gas motion can be described by solutions of other forms. For example, solutions of the form $y=f(\alpha t+\beta h)$, where $\alpha$ and $\beta$ are constants, can easily be found, and they describe steady motions. Solutions of the progressing wave type, similar to those considered by G. Guderley, by G. I. Taylor, by J. von Neumann and J. Calkin, and by R. Courant and K. O. Friedrichs (loc. cit., Chapter VI-C), can be obtained if $y$ has the form

$$
y=t^{a} f[K(h) t] .
$$

Here $a$ is a constant and $K(h)$ is a function of $h$ to be determined. By inserting (81) into (8) we find that $K(h)$ must have the form

$$
K(h)=(B h+C)^{1 /(1-\bullet)},
$$

where $B, C$ and $\epsilon$ are constants.

The resulting equation for $f$ can be reduced to first order, following von Neumann and Calkin, by introducing the new variables $s, F(s)$ and $q(F)$ defined by

$$
\begin{aligned}
s & =\log [K(h) t], \\
F(s) & =e^{-\delta s} f\left(e^{s}\right), \\
q(F) & =\left(\frac{d}{d s}+\delta\right) F(s) .
\end{aligned}
$$

In (83), the constant $\delta=[a n(1-\gamma)-2 a+2][2+n(\gamma-1)]^{-1}$. The equation (8) for $f$ now becomes the following equation for $q(F)$

$$
\begin{aligned}
& {\left[1-\gamma D F^{(n-1)(1-\gamma)} q^{-\gamma-1}\right](q-\delta F) q} \\
& =F^{(n-1)(1-\gamma)} q^{-\gamma} D\left[\gamma \epsilon+\gamma(n-1) q F^{-1}+\epsilon \gamma+a+1-\epsilon\right]-(a-1) a F-2 a q .
\end{aligned}
$$

In (84) $D$ is a constant. Special cases of (84) have been studied by von Neumann and Calkin.

Note added in proof. The solution (24)-(26) for $\gamma \neq 1$ and $\lambda \neq 0$, when specialized to the case $g_{0}=0$, coincides with the result of L. I. Sedov, "On the integration of the equations of one-dimensional gas motion", Doklady, AN USSR 90, 735 (1953). Some related results are given by M. L. Lidov, "Exact solution of the equations of one-dimensional unsteady gas motion taking into account Newtonian gravitational forces", Doklady, AN USSR 97, 409-410 (1954). 